\title{
Color Constancy based on the Grey-Edge Hypothesis
}

\author{
J. van de Weijer \\ Th. Gevers \\ Intelligent Sensory Information Systems \\ Faculty of Science, University of Amsterdam \\ Kruislaan 403, 1098 SJ Amsterdam, The Netherlands \\ \{joostw, gevers\}@science.uva.nl
}

\begin{abstract}
A well-known color constancy method is based on the GreyWorld assumption i.e. the average reflectance of surfaces in the world is achromatic. In this article we propose a new hypothesis for color constancy, namely the Grey-Edge hypothesis assuming that the average edge difference in a scene is achromatic. Based on this hypothesis, we propose an algorithm for color constancy.

Recently, the Grey-World hypothesis and the max-RGB method were shown to be two instantiations of a Minkowski norm based color constancy method. Similarly we also propose a more generale version of the Grey-Edge hypothesis which assumes that the Minkowsky norm of derivatives of the reflectance of surfaces is achromatic. The algorithms are tested on a large data set of images under different illuminants, and the results show that the new method outperforms the Grey-World assumption and the max-RGB method. Results are comparable to more elaborate algorithms, however at lower computational costs.
\end{abstract}

\section{Introduction}

Color constancy is the ability to recognize colors of objects invariant of the color of the light source [1], [2] [6]. It generally consists of two steps. Firstly, the light source color is estimated from the image data. Secondly, illuminant invariant descriptors are computed, which is usually done by adjusting the image for the color of the light source such that the object colors resemble the colors of the objects under a known light source.

A simple color constancy method, called max-RGB, estimates the light source color from the maximum response of the different color channels [1]. Another well-known color constancy method is based on the Grey-World hypothesis [4], which assumes that the average reflectance in the scene is achromatic. Although more elaborate algorithms exists, methods like Grey-World and max-RGB are still widely used because of their low computational costs.
Recently, Finlayson and Trezzi [5] showed that the maxRGB method and the Grey-World method can be interpreted as the same algorithm applied with different instantiations of the error function. The max-RGB method is shown to be equal to applying the $L^{\infty}$ Minkowski norm and GreyWorld is equal to using the $L^{1}$ norm. They further show that the best color constancy results are attained with the $L^{6}$ norm. Although these simple color constancy algorithms are slightly outperformed by more elaborate methods, e.g. color gamut mapping (for an overview see [1] [2]), they perform surprisingly well while their computational costs are significantly lower.

In this paper, we pursue color constancy by the GreyEdge hypothesis, which assumes the average edge difference in the scene to be achromatic. The method is based on the observation that the distribution of color derivatives exhibit the largest variation in the light source direction. The average of these derivatives is used to approximate this direction. The method is tested on a large database of colorful objects under varying lighting conditions and different illuminants. We further extend the method similarly to [5] and also derive color constancy for the error based on the various Minkowski norms.

The paper is organized as follows. In section 2 color constancy based on the Grey-World hypothesis is explained. In section 3 we propose the Grey-Edge hypothesis for color constancy computation. Section 4 contains experiments and Section 5 finishes with concluding remarks.

\section{The Grey-World Hypothesis}

The image values, $\mathbf{f}=(R, G, B)^{T}$, for a Lambertian surface are dependent on the light source $e(\lambda)$, where $\lambda$ is the wavelength, the surface reflectance $s(\lambda)$ and the camera sensitivity functions $\mathbf{c}(\lambda)=(R(\lambda), G(\lambda), B(\lambda))$

$$
\mathbf{f}=\int_{\omega} e(\lambda) s(\lambda) \mathbf{c}(\lambda) d \lambda
$$



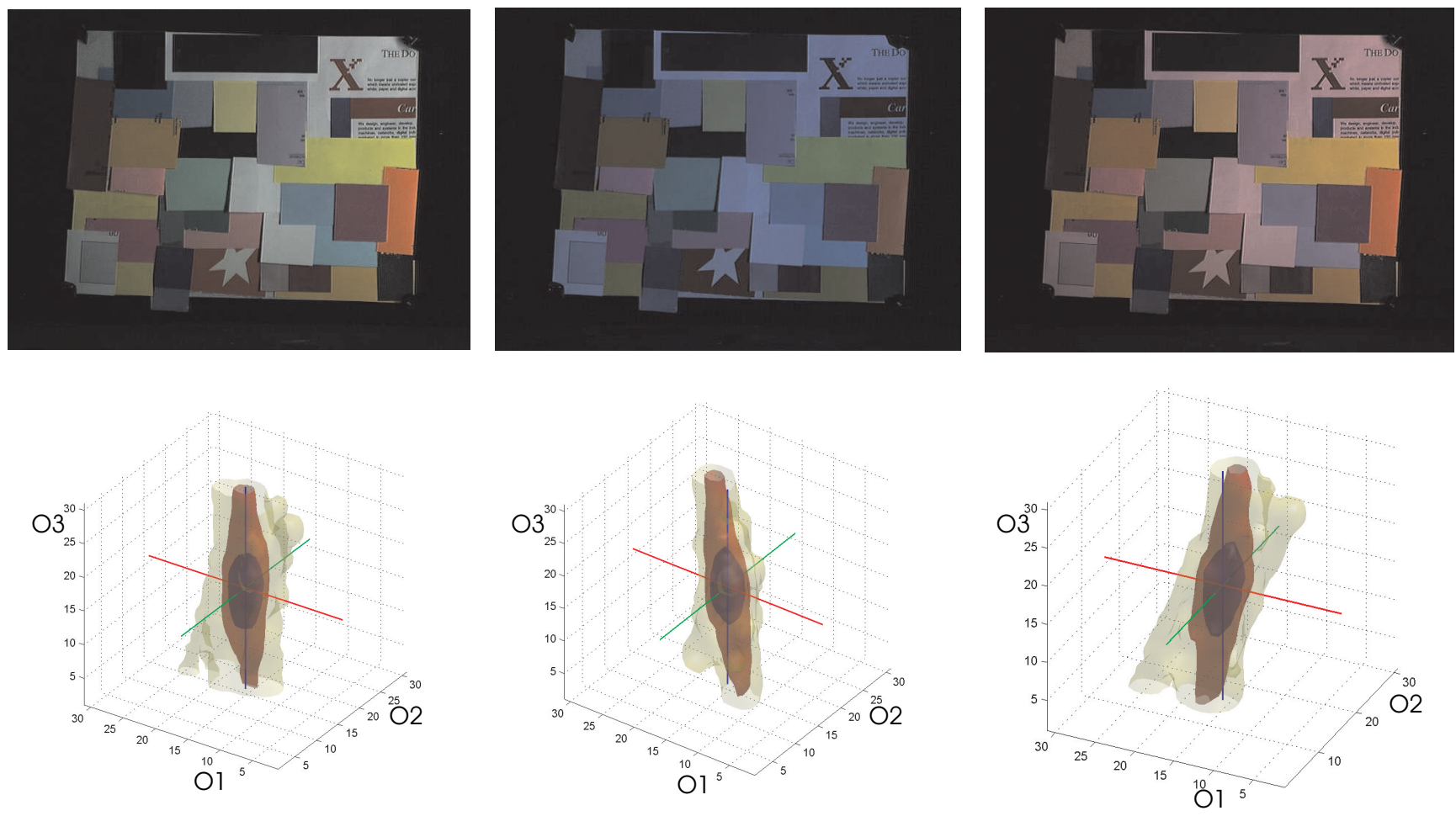

Figure 1: Three acquisitions of the same scene under different light sources [3]. On the bottom line the derivative distributions, where the axes are the opponent color derivatives and the surfaces indicate derivative values with equal occurrence and darker surfaces indicating a more dense distribution. Note the shift of the orientation of the distribution of the derivatives with the changing of the light source.

where $\omega$ is the visible spectrum and bold fonts are applied for vectors. The goal of color constancy is to estimate the light source color $e(\lambda)$, or its projection on the $R G B$ kernels,

$$
\mathbf{e}=\left(\begin{array}{c}
R_{e} \\
G_{e} \\
B_{e}
\end{array}\right)=\int_{\omega} e(\lambda) \mathbf{c}(\lambda) d \lambda
$$

given the image values $\mathbf{f}(\mathbf{x})$, where $\mathbf{x}$ is the spatial coordinate in the image. The task of color constancy is not attainable without further assumptions.

Buchsbaum [4] proposes the Grey-World hypothesis, which assumes that the average reflectance in a scene is achromatic:

$$
\frac{\int s(\lambda, \mathbf{x}) d \mathbf{x}}{\int d \mathbf{x}}=k .
$$

The light source color can now be estimated by computing the average pixel value, since

$$
\begin{aligned}
\frac{\int \mathbf{f}(\mathbf{x}) d \mathbf{x}}{\int d \mathbf{x}} & =\frac{1}{\int d \mathbf{x}} \iint_{\omega} e(\lambda) s(\lambda, \mathbf{x}) \mathbf{c}(\lambda) d \lambda d \mathbf{x} \\
& =k \int_{\omega} e(\lambda) \mathbf{c}(\lambda) d \lambda=k \mathbf{e}
\end{aligned}
$$

which yields the normalized light source color :ê $=$ $k \mathbf{e} /|k \mathbf{e}|$. This is indeed a very simple algorithm to find the light source color of a scene.

In [5] it is shown that the Grey-World hypothesis can be improved by replacing the averaging operation by the Minkowski norm. In this case Eq. 4 can be rewritten as

$$
\left(\frac{\int \mathbf{f}^{p}(\mathbf{x}) d x}{\int d x}\right)^{\frac{1}{p}}=k \mathbf{e}
$$

For $p=1$ the equation is equal to the Grey-World assumption. For $p=\infty$ it is equal to color constancy by max-RGB, which is based on the assumption that the maximum response in the channels is caused by a white patch. Hence, the maximum responses yield an estimate of the light source. Finlayson and Trezzi [5] found that the best results are obtained with a Minkowski norm with $p=6$.

\section{The Grey-Edge Hypothesis}

As an alternative to the Grey-World hypothesis, we propose the Grey-Edge hypothesis; the average of the reflectance 

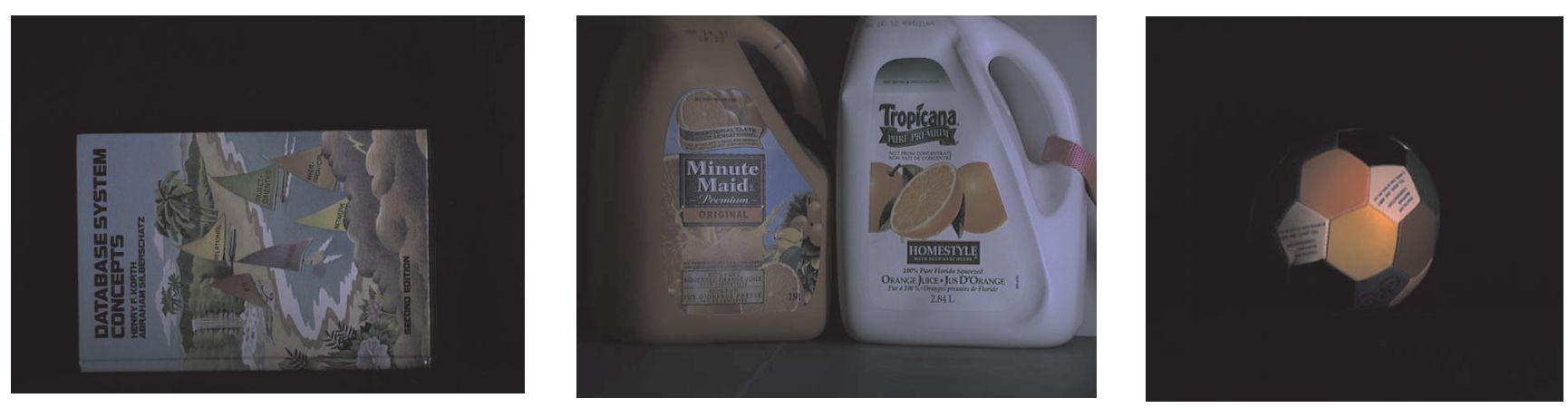

Figure 2: Examples of the images in group $A$ and B [3].

differences in a scene is achromatic

$$
\frac{\int\left|s_{x}(\lambda, x)\right| d x}{\int d x}=k
$$

With the Grey-Edge assumption the light source color can be computed from the average color derivative in the image given by:

$$
\begin{gathered}
\frac{\int\left|\mathbf{f}_{\mathbf{x}}(\mathbf{x})\right| d \mathbf{x}}{\int d \mathbf{x}}=\frac{1}{\int d \mathbf{x}} \iint_{\omega} e(\lambda)\left|s_{\mathbf{x}}(\lambda, \mathbf{x})\right| \mathbf{c}(\lambda) d \lambda d \mathbf{x} \\
=k \int_{\omega} e(\lambda) \mathbf{c}(\lambda) d \lambda=k \mathbf{e},
\end{gathered}
$$

where $\left|\mathbf{f}_{\mathbf{x}}(\mathbf{x})\right|=\left(\left|R_{\mathbf{x}}(\mathbf{x})\right|,\left|G_{\mathbf{x}}(\mathbf{x})\right|,\left|B_{\mathbf{x}}(\mathbf{x})\right|\right)^{T}$. The Grey-Edge hypothesis originates from the observation that the color derivative distribution of images forms a relatively regular, ellipsoid-like shape, of which the long axis coincides with the light source color. In Fig. 1 the color derivative distribution is depicted for three images. The color derivatives are rotated to the opponent color space

$$
\begin{aligned}
& O 1_{\mathbf{x}}=\frac{R_{\mathbf{x}}-G_{\mathbf{x}}}{\sqrt{2}} \\
& O 2_{\mathbf{x}}=\frac{R_{\mathbf{x}}+G_{\mathbf{x}}-2 B_{\mathbf{x}}}{\sqrt{6}} . \\
& O 3_{\mathbf{x}}=\frac{R_{\mathbf{x}}+G_{\mathbf{x}}+B_{\mathbf{x}}}{\sqrt{3}}
\end{aligned}
$$

In the opponent color space, $O 3$ coincides with the white light direction. For the scene under white light (the leftmost picture) the distribution of the derivatives are centered along the $O 3$ or white-light axis. Once we change the color of the light source as in the second and third picture, the distribution of the color derivatives no longer align with the white-light axis. Color constancy based on the Grey-Edge assumption can be interpreted as skewing the color derivative distribution such that the average derivative is in the $O 3$ orientation.

Similarly as for the Grey-World based color constancy, the Grey-Edge hypothesis can also be adapted to incorpo- rate the Minkowsky norm

$$
\left(\frac{\int\left|\mathbf{f}_{\mathbf{x}}(\mathbf{x})\right|^{p} d \mathbf{x}}{\int d \mathbf{x}}\right)^{\frac{1}{p}}=k \mathbf{e} .
$$

Color constancy based on this equation assumes that the $p$ th Minkowski norm of the derivative of the reflectance in a scene is achromatic.

\section{Experiments}

To test the Grey-Edge hypothesis the algorithm is tested on a large data set of colorful object under varying light sources [3]. The data set is split in two groups. Group A consists of 321 images with varying light sources over a total of 32 scenes and group B consists of 220 images of 22 scenes (see examples in Fig. 2). For all images the correct light source is measured, $\mathbf{e}_{l}$. As an error measure we use the angular error between the the estimated light source $\mathbf{e}_{e}$ and the measured light source $\mathbf{e}_{l}$

$$
\text { angular error }=\cos ^{-1}\left(\hat{\mathbf{e}}_{l} \cdot \hat{\mathbf{e}}_{e}\right),
$$

where the (^) indicates the normalized vector. Results of other color constancy algorithms on this standard data set are available in [2], [7], [5]. For the derivatives Gaussian derivatives with $\sigma=3$ were applied.

In Fig. 3 the results for the Grey-World and the GreyEdge assumption as a function of the applied norm, $p$, are depicted. The results of the Grey-World are taken from [5]. The angular error for the Grey-Edge method outperforms the Grey-World method for both groups of images. Whereas the Grey-World method finds a minimum error for the same norm, $p=6$ for both groups of images, for the Grey-Edge method the behavior as a function of $p$ varies for the two groups of images. If we compare $p=6$ for the Grey-World with $p=16$ for the Grey-Edge based method, 

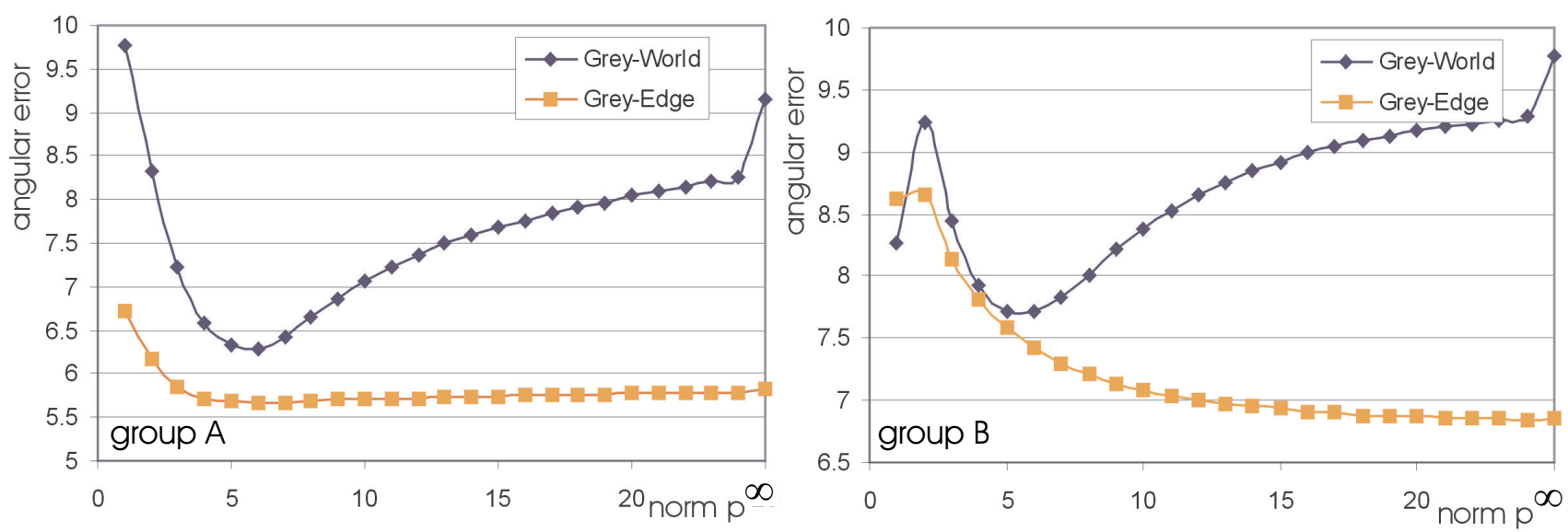

Figure 3: Angular error of the Grey-World and the Grey-Edge method as a function of the applied Minkowski norm.

\begin{tabular}{|l|c|}
\hline & Mean \\
\hline Grey-World (=L $L^{1}$-norm) & 9.8 \\
\hline Max-RGB $\left(=L^{\infty}\right.$-norm) & 9.2 \\
\hline L6-norm Grey-World & 6.3 \\
\hline$L 6$-norm Grey-Edge & 5.7 \\
\hline Color by Correlation & 9.9 \\
\hline Gamut Mapping & 5.6 \\
\hline GCIE Version 3, 11 lights & 4.9 \\
\hline
\end{tabular}

\section{Conclusions}

In this paper we proposed a color constancy algorithm based on the Grey-Edge hypothesis which assumes the average edge difference in a scene to be achromatic. Further, an extension based on the Minkowski norm is proposed. The algorithm is tested on a large data set and is shown to outperform color-constancy based on the Grey-World hypothesis and the max-RGB assumption.

Table 1: Mean angular error (degrees) for various color constancy methods on group A images [7].

we attain an improvement of $9 \%$ for the images in group A and of $10 \%$ for the images in group B.

Also the $p=\infty$ norm, which is the Grey-Edge variant on the max-RGB method, achieves a good performance. The light source is computed from the assumption that the light source is equal to the maximum derivatives of the various color channels.

Results of more complex color constancy methods, such as gamut mapping and color-by-correlation, have been reported in [2], [7] for the images in group A. The results are comparable to the results reported here and only two methods perform slightly better, see Table 1 . For example for Gamut mapping an angular error of $5.6^{\circ}$ was reported (opposed to $5.7^{\circ}$ for the Grey-Edge based color constancy). These methods are, however, considerably more complex and therefore require higher computational costs. In conclusion, the presented Grey-Edge method is an useful alternative when computational speed is an issue, with a performance comparable to the best results reported in literature.

\section{References}

[1] K. Barnard, V. Cardei, and B.V. Funt. A comparison of computational color constancy algorithms-part i: Methodology and experiments with synthesized data. IEEE transactions on Image Processing, 11(9):972-984, September 2002.

[2] K. Barnard, V. Cardei, and B.V. Funt. A comparison of computational color constancy algorithms-part ii: Experiments with image data. IEEE transactions on Image Processing, 11(9):985-996, September 2002.

[3] K. Barnard, L. Martin, B.V. Funt, and A. Coath. A data set for colour research. Color Research and Application, 27(3):147151, 2002.

[4] G. Buchsbaum. A spatial processor model for object colour perception. Journal of the Franklin Institute, 310, 1980.

[5] G.D. Finlayson and E. Trezzi. Shades of gray and colour constancy. In IS\&T/SID Twelfth Color Imageing Conference, pages 37-41, 2004.

[6] D.A. Forsyth. A novel algorithm for color constancy. International Journal of Computer Vision, 5(1):5-36, 1990.

[7] S.D. Hordley G.D. Finlayson and I. Tastl. Gamut constrained illuminant estimation. In Proc. of the Ninth IEEE International Conference on Computer Vision, Nice, France, 2003. 